\title{
José Zudaire*
}

Ángela Alférez**

\section{LA MADUREZ DEL VENTURE CAPITAL EN ESPAÑA}

En menos de una década y coincidiendo con el inicio de la última crisis económica, el venture capital o capital riesgo en España ha pasado de ser un segmento de inversión minoritario con escasas gestoras, rondas de inversión de tamaños muy pequeños y un papel especialmente destacado del inversor público, a configurarse como un ecosistema con numerosos actores (aceleradoras, business angels, corporates ventures, fondos de venture capital...) no solo públicos, también privados e internacionales, que con su intensa actividad han contribuido a través de diversas iniciativas a consolidar el capital riesgo nacional como uno de los más interesantes dentro del panorama europeo.

Palabras clave: private equity, startup, financiación no bancaria, hub, empleo, ecosistema emprendedor. Clasificación JEL: G23, G24, L26, M13.

\section{Introducción}

El venture capital en España se ha ido consolidando en los últimos años como una opción cada vez más sólida para la financiación a largo plazo de empresas en fase inicial (startups). En torno a la inversión en empresas no cotizadas se ha ido configurando así un ecosistema caracterizado por la variedad de agentes, iniciativas y políticas cuyo número crece exponencialmente y que están empujando su

\footnotetext{
* Director General de la Asociación Española de Capital, Crecimiento e Inversión (ASCRI).

** Directora de Estudios y Venture Capital (ASCRI).
}

protagonismo en la escena europea. Los inicios del venture capital ${ }^{1}$ se remontan a los años setenta con una iniciativa liderada exclusivamente por sociedades

\footnotetext{
1 La terminología en este sector varía en función del mercado del que se esté hablando. Los anglosajones han diferenciado prácticamente desde sus inicios los términos venture capital (inversión en empresas en fases iniciales o de desarrollo temprano) y private equity (inversión en empresas en fases de crecimiento y consolidadas). En España, por el contrario, en los comienzos de la implantación de este sector se utilizaba únicamente el término capital riesgo, ahora capital privado, y en general como equivalencia del término venture capital, ya que prácticamente no había inversiones en empresas maduras. Con el desarrollo del sector, se añadió al término capital riesgo el término private equity y así poder englobar todas las fases posibles de la inversión en una empresa (iniciales y maduras). Actualmente en España, y siguiendo la terminología anglosajona, se ha implantado la utilización de los dos términos venture capital y private equity dependiendo del tipo de inversión al que se haga referencia, o capital privado o capital riesgo si se quiere englobar a todo el conjunto del sector.
} 
de carácter público ${ }^{2}$, cuyo objetivo era contribuir al desarrollo de las regiones menos desfavorecidas a través de un instrumento que apoyase a la empresa, bajo una filosofía de inversión a largo plazo y con fórmulas en las que predominaba financiar a través del capital o semicapital frente a instrumentos de deuda. A pesar de que este sector cuenta con un período relativamente largo de vida desde sus inicios, el desarrollo del venture capital en España ha sido pequeño si se compara con el private equity. En ambos tipos de inversión su evolución ha estado muy ligada a las diferentes fases de los ciclos económicos y su impacto ha impulsado cambios que han ido configurando el sector del capital privado ${ }^{3}$ hasta lo que es hoy en día. En concreto, no fue hasta la crisis financiera iniciada en 2008 cuando el venture capital se ha consolidado como un sector que ha ganado en madurez y tamaño para promover el desarrollo del emprendimiento e innovación en España. En este artículo se pretende describir el desarrollo reciente del capital riesgo, analizar las características actuales que están marcando su dinámica y apuntar las diferentes medidas que sería necesario promover para impulsar al sector como uno de los líderes del entorno europeo.

\footnotetext{
2 La Sociedad para el Desarrollo Industrial de Galicia (SODIGA) fue la primera entidad con capital mayoritariamente público que inició su actividad en 1972 para promover la actividad económica a través de la participación temporal y minoritaria en el capital de empresas como herramienta para la promoción empresarial.

3 Las características que configuran el capital privado como uno de los principales instrumentos de intermediación financiera no bancaria, y en concreto el principal mecanismo para proporcionar recursos propios a las empresas no cotizadas, se pueden resumir en los siguientes puntos:

- La existencia de un inversor especializado (entidad de capital privado que puede tener carácter público o privado) que, además de recursos, aporta capacidad de gestión y creación de valor a la empresa participada.

- La aportación de recursos a la empresa se hace, normalmente, a través de suscripción de acciones, aunque también se pueden utilizar otros instrumentos financieros, tales como los préstamos participativos, préstamos subordinados o suscripción de obligaciones convertibles.

- Las adquisiciones que llevan a cabo las entidades de capital privado tienen un carácter temporal (entre tres y siete años).

- Los retornos se materializan una vez finalizado el período de inversión a través de un mecanismo de salida, que puede estructurarse mediante venta a otras empresas, colocación en el mercado de valores, venta a otra entidad de capital riesgo o venta al propio equipo directivo o a los accionistas originales.
}

\section{Evolución reciente del venture capital}

La evolución de la inversión del venture capital en startups españolas refleja la madurez que el mercado está alcanzando en los últimos años. El año 2013 registró el mínimo histórico con 194.000.000 de euros de inversión; desde entonces se han alcanzado los mejores registros desde que se elaboran estadísticas específicas de venture capital, tal y como se puede ver en el Gráfico 1.

En concreto, en 2017 (último año del que se tienen estadísticas) el volumen de inversión superó los 537.700.000 euros repartidos en 560 inversiones (449 empresas), situando este ejercicio como uno de los mejores por detrás de 2015, en el que se alcanzó un máximo histórico (548.000.000 de euros). Detrás de estas cifras participan tres tipos de entidades cuyo papel ha ido variando desde la perspectiva temporal y que actualmente, gracias a su foco y estrategia de inversión, cubren gran parte de las necesidades de financiación no bancaria de empresas en primeras fases.

\section{Entidades nacionales públicas}

Fueron las protagonistas en la implantación y desarrollo del venture capital y del private equity en España, destacando el papel que han ejercido para impulsar el tejido empresarial en aquellas zonas más desfavorecidas y en fases de la empresa donde es más complicado canalizar capital privado 4 . Su actividad, impulsada por organismos regionales o estatales, se ha apoyado fundamentalmente en instrumentos como la subvención (al inicio de su actividad) y más recientemente el equity $\mathrm{y}$, fundamentalmente, el semi equity a través de los préstamos participativos. El enfoque inversor de estas entidades públicas inicialmente se canalizaba hacia empresas pequeñas, generalmente de ámbito regional, y en

\footnotetext{
4 La primera experiencia cercana a lo que conocemos como capital privado se remonta a la creación de la Sociedad para el Desarrollo Industrial de Galicia, con capital mayoritariamente público, en 1972.
} 


\section{GRÁFICO 1}

\section{EVOLUCIÓN DE LA INVERSIÓN DE CAPITAL RIESGO SEGÚN TIPO DE ENTIDAD EN EMPRESAS ESPAÑOLAS, 2006-2017}

(En millones de euros)

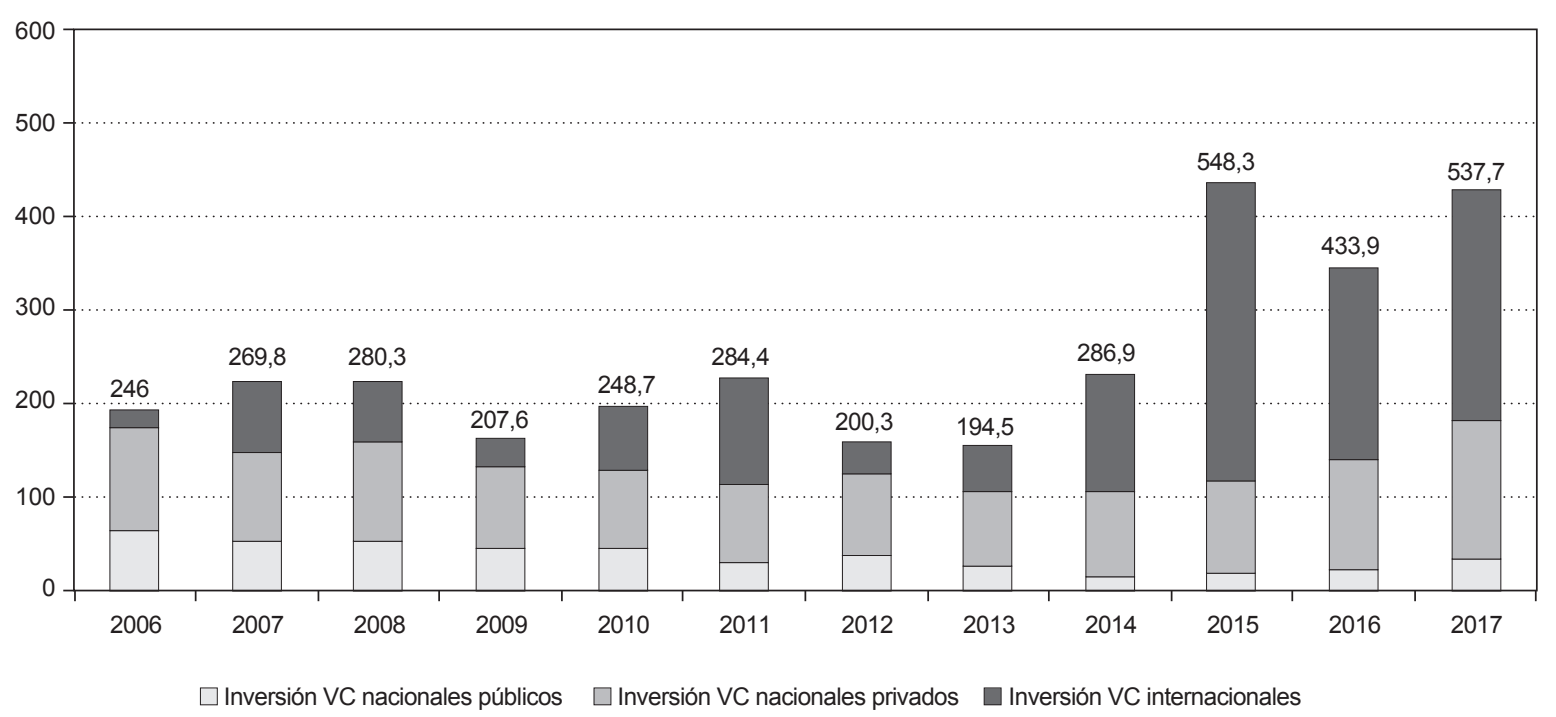

FUENTE: ASCRI/webcapitalriesgo. https://www.ascri.org/

sectores con bajo contenido tecnológico. A partir de la crisis iniciada en 2008 , las sociedades públicas con actividad en el segmento de venture capital fueron replegando su actividad como financiador directo de las empresas debido al ajuste presupuestario que experimentó la Administración. No obstante, a nivel estatal su aportación a la financiación de la startup española se reorientó a través de su actividad como inversor en fondos de venture capital (limited partner en terminología anglosajona) con programas de fondos como Innvierte, que lideró CDTI, y sobre todo Fond-ICO Global, gestionado por Axis/ICO, convirtiéndose en inversores ancla de muchos de los nuevos fondos de venture capital lanzados recientemente. Esta actividad ha incidido muy positivamente en la dotación de recursos a los fondos de capital riesgo nacionales privados orientados a la inversión en proyectos de transferencia de tecnología e innovación. En 2017 las entidades de capital riesgo públicas recuperaron parte del terreno perdido en la inversión directa, doblando los volúmenes registrados en 2016 , al situarse la actividad inversora en 44.000 .000 de euros, repartidos en 152 inversiones (Gráfico 2). Este volumen representó solo el 8 por 100 del total de la inversión en venture capital en España en 2017 y, aunque su peso es pequeño en términos de volumen, juegan un papel determinante en la financiación de empresas fuera de los dos centros principales de innovación (Madrid y Barcelona) y en sectores de corte tradicional.

Actualmente, en el mercado español hay unas 19 entidades de capital privado públicas sin una orientación inversora específica, si bien la mayor parte de ellas (en torno a 15) canalizan parte de su actividad a la financiación de proyectos que se encuentran en las fases más tempranas de las startups. El ticket medio de inversión 


\section{GRÁFICO 2}

\section{EVOLUCIÓN DE LA INVERSIÓN DE VENTURE CAPITAL DE LAS ENTIDADES PÚBLICAS EN EMPRESAS ESPAÑOLAS, 2013-2017}

(En millones de euros)

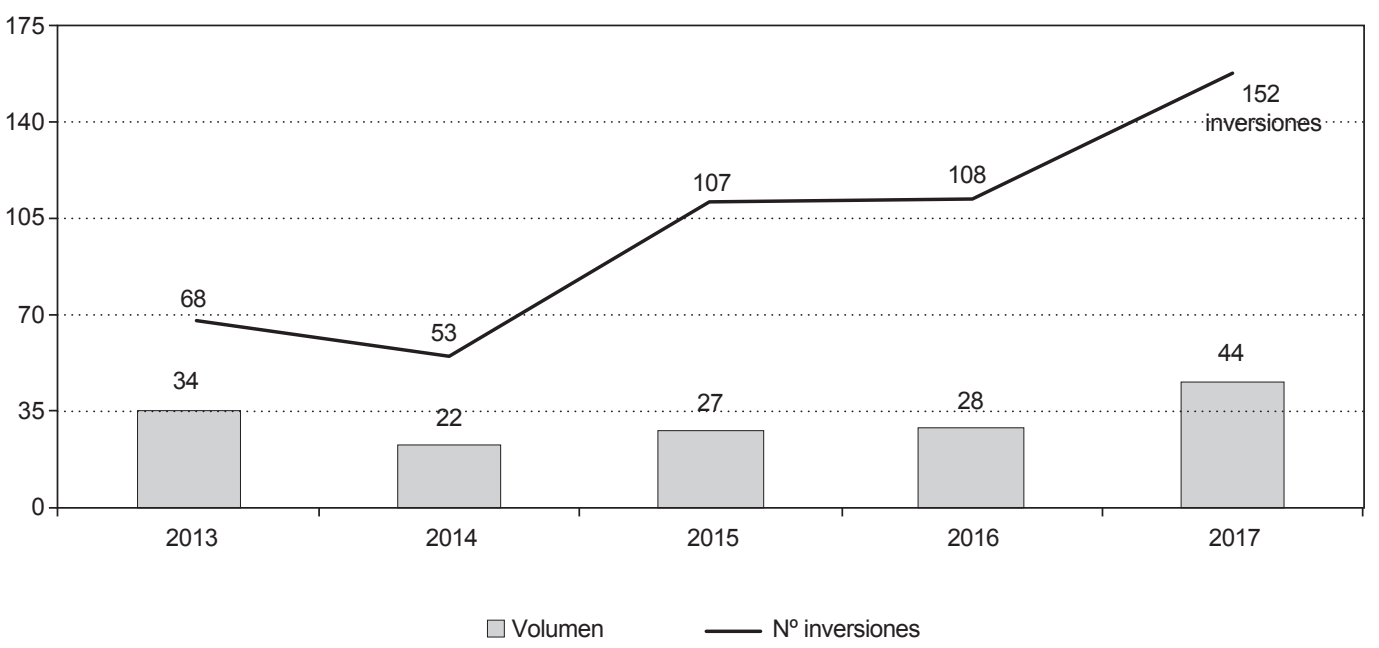

FUENTE: ASCRI/webcapitalriesgo.

por proyecto de las gestoras públicas entre 2015 y 2017 fue de 266.000 euros.

\section{Gestoras nacionales privadas}

La aparición en escena de las entidades privadas nacionales como agentes financiadores de la empresa española se produjo a partir de la entrada de España en la Unión Europea en 1986. En sus inicios, la actividad de este tipo de gestoras estaba orientada fundamentalmente a la inversión en empresas en fases maduras (private equity). A partir de finales de los años noventa, paulatinamente empezaron a surgir nuevos fondos con un enfoque dirigido a la inversión en empresas emergentes, aunque su aparición fue muy marginal. Algunas de estas entidades se vieron afectadas, además, por las dos recesiones que han impactado en el sector del venture capital, y desaparecieron, ocupando su lugar otras ante las expectativas abiertas por las nuevas tecnologías.
Tal y como describe Trenchs (2018), las gestoras de venture capital nacionales se pueden dividir en tres tipos:

- Gestoras que empezaron a operar en los primeros años de inicio del venture capital en España (Bullnet Gestión, Adara Ventures, Nauta Capital VC Partners, Inveready Technology Investment Group, Axon Partners Group, Uninvest, Grupo Intercom o Caixa Capital Risc, entre otros) y apostaron por un mercado con escasa oferta e inmaduro. Las gestoras que han sobrevivido cuentan hoy en día con tres o más fondos abiertos, síntoma claro de su madurez.

- Gestoras que comenzaron entre 2008 y 2013, en plena crisis financiera, cuya puesta en marcha contaba con un perfil especialista en sectores como los de ciencias de la vida, digital, etc. (Ysios Capital Partners, Kibo Ventures, Swanlaab, CRB Inversiones Biotecnológicas o Seaya Ventures son algunos de los ejemplos).

- Gestoras creadas tras la crisis financiera de 2008 por emprendedores de éxito que han tenido experiencias 


\section{GRÁFICO 3}

\section{EVOLUCIÓN DE LA INVERSIÓN DE VENTURE CAPITAL DE LAS ENTIDADES PRIVADAS NACIONALES EN EMPRESAS ESPAÑOLAS, 2013-2017 \\ (En millones de euros)}

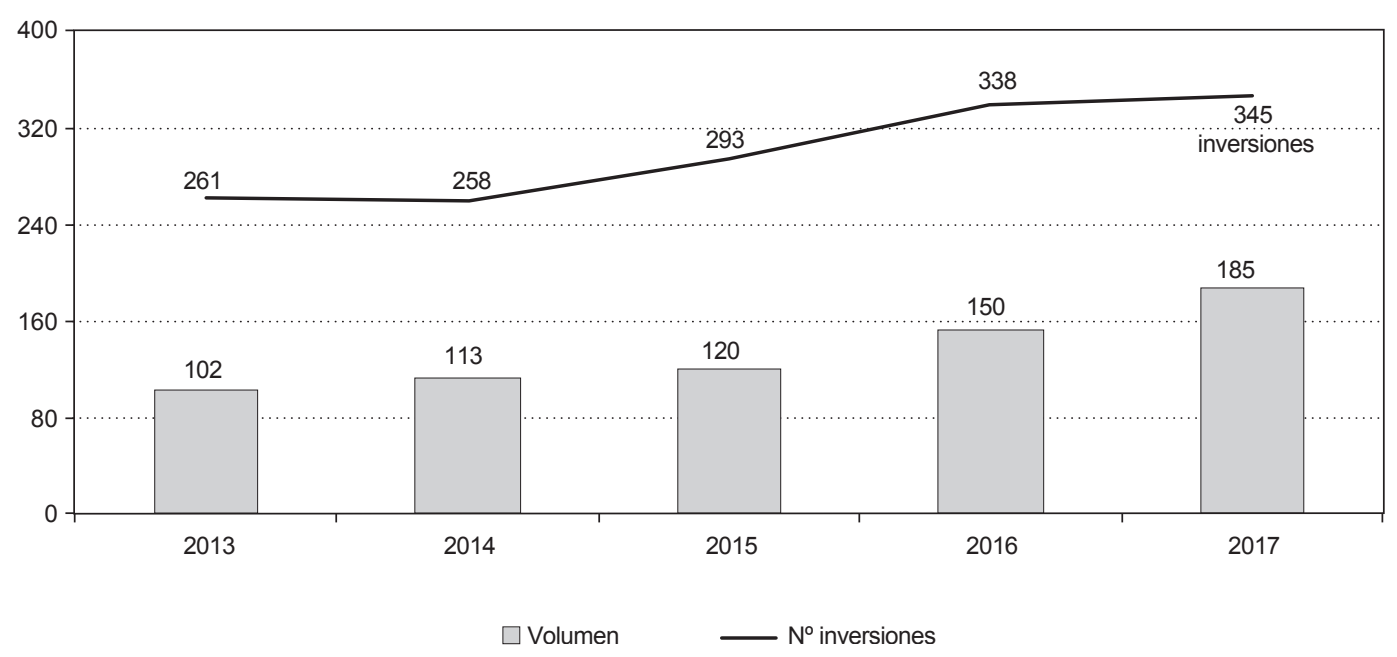

FUENTE: ASCRI/webcapitalriesgo.

positivas en su etapa de fundadores de empresas y que deciden levantar un fondo para repetir el proceso con nuevas iniciativas, como Columbus, Samaipata Capital Partners, KFund o Seed Rocket 4Founders Capital.

La captación de recursos llevada a cabo por las gestoras nacionales privadas en los últimos años, así como la aparición de nuevos operadores, han dotado de capacidad inversora a estos fondos y su contribución a la financiación de startups ha crecido, tanto en términos de volumen como proyectos financiados. Hay contabilizadas cerca de 90 entidades de venture capital nacionales privadas que en los últimos dos años están aportando en torno al 34 por 100 del total de la inversión de venture capital anual en España. Su aportación al desarrollo del sector en España destaca en el número de proyectos financiados cuya media, en los últimos dos años, está en torno al 65 por 100 , la mayor parte en fases semilla ${ }^{5} y$

\footnotetext{
5 Inversión dirigida a empresas que no tienen ventas.
}

arranque $^{6}$. En concreto, en 2017 las entidades de venture capital nacionales privadas potenciaron la inversión en primeras fases en España, tanto en volúmenes invertidos (185.200.000 euros) como número de inversiones (345), registrando máximos históricos en ambas variables (Gráfico 3).

\section{Gestoras internacionales}

La aparición de las gestoras internacionales en el mercado español se produjo a principios de la década de los años noventa, con una orientación inversora dirigida a empresas consolidadas. Desde entonces, su presencia ha sido creciente, especialmente a partir de 2011 cuando las gestoras de venture capital internacionales comenzaron a poner el foco en las startups españolas.

\footnotetext{
6 La inversión en fase arranque o startup se dirige a empresas con ventas pero EBITDA negativo.
} 
En los últimos años se están detectando varias tendencias en relación a las gestoras internacionales de venture capital con actividad en España:

- Crecimiento anual del número de inversiones cerradas por estos fondos. En 2017 cerraron el máximo histórico de inversiones en empresas emergentes españolas (63 en 34 empresas), síntoma de la confianza y madurez en el mercado español.

- Crecimiento exponencial de nuevos fondos internacionales de venture capital con actividad en España. En 2017, de las 26 nuevas gestoras que realizaron una inversión por primera vez en el mercado español, 18 fueron de venture capital. No obstante, por el momento ninguna de estas gestoras ha abierto oficina en España. A finales de 2017, un total de 118 fondos internacionales contaban en su cartera con alguna startup española.

- Al igual que está sucediendo con el private equity, las gestoras internacionales de venture capital están dotando de tamaño a este segmento de inversión gracias a unos tickets de inversión de tamaño superior a los de las gestoras nacionales, cubriendo el gap de financiación que, por el momento, los fondos nacionales no son capaces de abarcar debido al tamaño de sus fondos. En este sentido, a partir de 2014, y gracias a estos fondos, en el mercado español se empezaron a ver rondas superiores a los 10.000 .000 de euros dirigidas a startups, y en 2016 se cerró la primera operación por encima de los 100.000.000 de euros. En 2017 se contabilizaron cinco inversiones por encima de los 10.000.000 de euros, de las cuales una superó de nuevo los 100.000 .000 de euros, estando todas estas rondas protagonizadas por fondos internacionales.

- Foco de inversión en proyectos relacionados con el sector digital y consumo. El 80 por 100 del volumen de inversión que recibió esta categoría en 2017 (307.600.000 euros) provino de fondos de venture capital internacionales, aunque el interés de estos fondos por el sector ciencias de la vida/biotecnología está creciendo en los últimos años (en 2017 aportaron 31.000 .000 de euros de los 66.900 .000 que recibió el sector para financiar proyectos de venture capital).
- La mayor parte de la financiación se dirige a startups en fase avanzada (late stage venture ${ }^{7}$ y other early stages ${ }^{8}$ ), normalmente en coinversión con gestoras nacionales, aunque cada vez es más común ver sindicatos de gestoras internacionales sin presencia de inversor local.

Los fondos internacionales están aportando en torno al 60 por 100 del total invertido en venture capital en España. En concreto, en 2017 su inversión alcanzó los 308.000.000 de euros dirigidos a startups nacionales (Gráfico 4 y Cuadro 1).

Sin duda, 2017 ha sido un ejercicio muy positivo para el venture capital ya que se trasluce el apetito inversor que despierta este mercado (300 startups españolas recibieron financiación de venture capital por primera vez, mejor registro histórico). Entre los factores que están marcando la madurez de este segmento de inversión destacan la presencia, cada vez más intensa, de los fondos internacionales (que complementa el reciente dinamismo de las gestoras privadas españolas) y una intensificación de la actividad en segmentos de mercado que hasta ahora no estaban cubiertos, como el comprendido entre los tramos de 5.000 .000 a 10.000 .000 de euros. Así, en 2017 se cerraron 15 inversiones en ocho empresas, y 88 scaleups consiguieron inversión por encima de 1.000.000 de euros, doblando el registro de 2012.

\section{Principales características del ecosistema}

El desarrollo de la inversión de venture capital está estrechamente relacionado con el ecosistema que se forma en torno a este tipo de inversión. Numerosos países pretenden replicar experiencias como Silicon Valley o Israel, por ser ejemplos claros de ecosistemas de éxito. Sin embargo, un análisis más profundo de las razones por las que ambos lugares han llegado a convertirse en modelos de referencia revela que hay

\footnotetext{
7 Inversión dirigida a pymes en crecimiento con ventas y EBITDA positivo.

8 Rondas de follow on o seguimiento en una empresa que ya ha recibido financiación de venture capital anteriormente.
} 


\section{GRÁFICO 4}

\section{EVOLUCIÓN DE LA INVERSIÓN DE VENTURE CAPITAL DE LAS ENTIDADES INTERNACIONALES EN EMPRESAS ESPAÑOLAS, 2013-2017}

(En millones de euros)

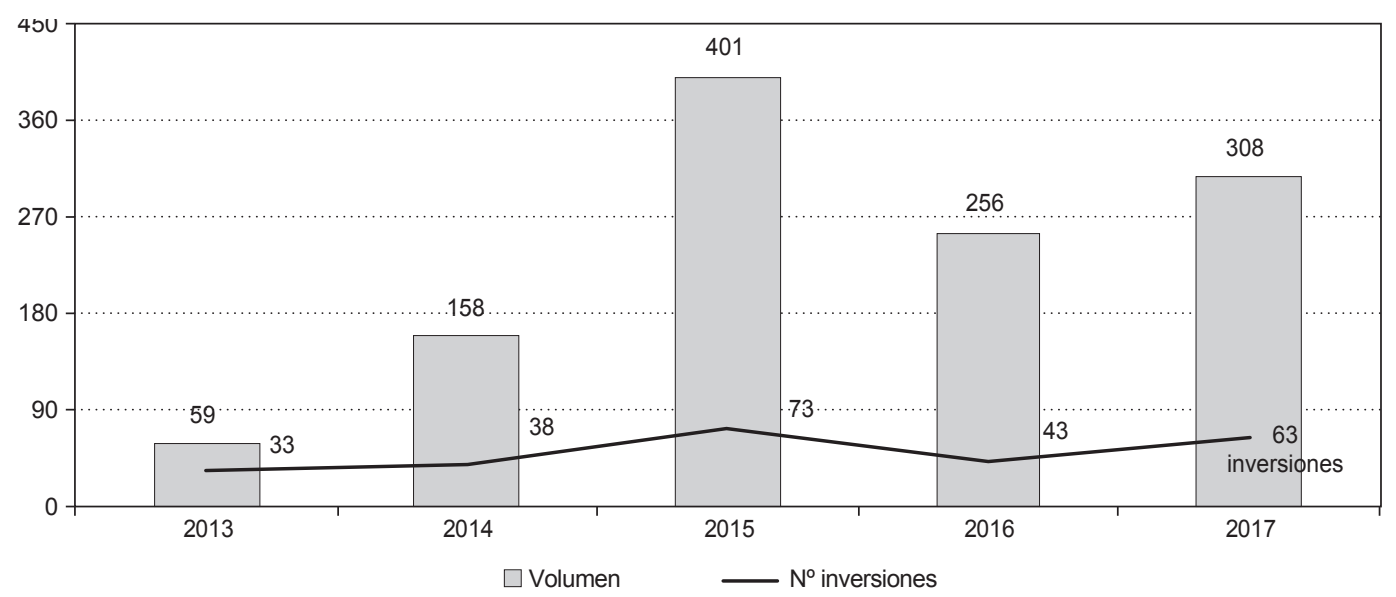

FUENTE: ASCRI/webcapitalriesgo.

\section{CUADRO 1}

\section{RESUMEN DE LA INVERSIÓN DE VENTURE CAPITAL EN STARTUPS ESPAÑOLAS EN 2017} (En millones de euros)

VC (inversión).

VC Público (inversión)

№ de inversores activos 2017 


\section{GRÁFICO 5}

EVOLUCIÓN DE LA INVERSIÓN DE ACELERADORAS, CROWDFUNDING, INCUBADORAS Y REDES DE BUSINESS ANGELS DE LAS ENTIDADES INTERNACIONALES EN EMPRESAS ESPAÑOLAS, 2006-2017

(En millones de euros)

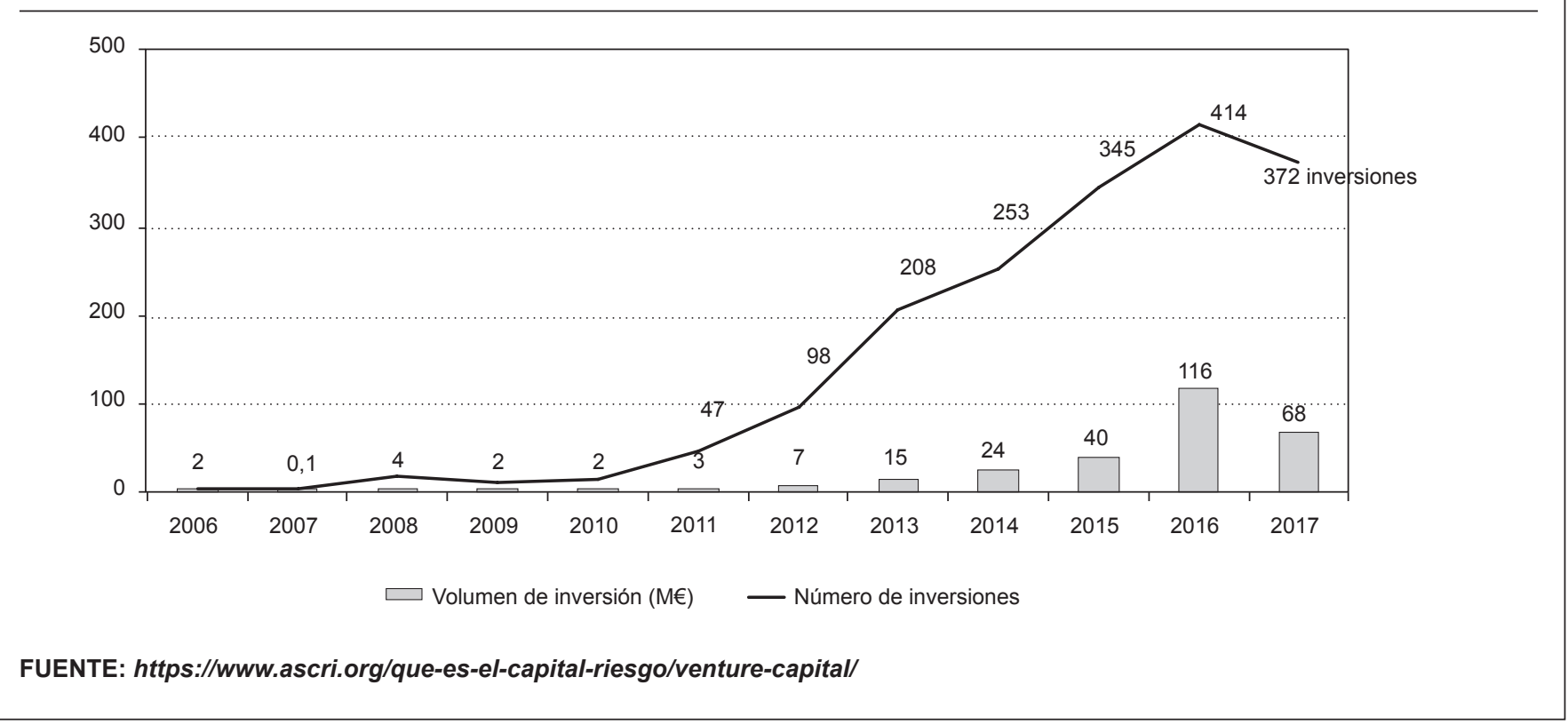

circunstancias difícilmente replicables, por lo que cada país debe tratar de sacar su potencial para conseguir posicionarse como un país innovador.

En el caso de España, a partir de la crisis iniciada en 2008, se está configurando un ecosistema que cada año crece en número de iniciativas y con unas características que se pueden resumir en las siguientes:

- Consolidación de las redes de business angels, aceleradoras e incubadoras que, gracias a su actividad, están impulsando la financiación de empresas vinculadas a todo tipo de sectores en casi todas las regiones españolas y en las fases más iniciales del proyecto. Complementando la actividad inversora, en los últimos años son numerosas las iniciativas que se han puesto en marcha tales como foros de inversión, talleres, formación etc., orientadas a promover el emprendimiento. En 2017 estos inversores canalizaron un total de 68.000 .000 de euros en empresas españolas, segundo mejor registro por detrás de 2016, en el que invirtieron 116.000 .000 de euros (máximo histórico que multiplicó por cinco la cifra alcanzada tan solo dos años antes) (Gráfico 5).

- Presencia de las grandes corporaciones, piezas clave para el desarrollo del ecosistema y de la actividad de venture capital. La atención de las grandes corporaciones españolas al fenómeno startup, creando programas o vehículos de inversión específicos que les permita el desarrollo de proyectos innovadores relacionados con su actividad principal, está ganando protagonismo en España. Telefónica, Repsol e Iberdrola fueron de las primeras grandes empresas españolas que crearon vehículos de corporate venture dentro de sus organizaciones, así como programas de aceleración de startups. En los últimos tres años otras empresas están apostando por esta línea de negocio (Santander, BBVA, Indra, Prosegur, Amadeus, Ferrovial, Seat o Cuatrecasas, entre otras) e incluso formando conglomerados, como es 
la puesta en marcha de la iniciativa Start4Big, hub que CaixaBank, Seat, Telefónica, Agbar y Naturgy han lanzado para atraer innovación. No obstante, por el momento su integración en el ecosistema no es tan fuerte como en otros países en los que el corporate venture está muy desarrollado y tiene una amplia presencia, tal y como sucede en Israel o Reino Unido. La actividad de los corporate venture nacionales se complementa con los corporate internacionales, que progresivamente están ganando en interés hacia el mercado español, protagonizando, como en el caso de Rakuten, la mayor ronda de financiación de una startup en España. En 2017 la inversión de los corporate internacionales en startups españolas superó los 146.700.000 euros, frente a los 8.700 .000 euros invertidos por los corporate venture nacionales ${ }^{9}$.

- Apoyo de las instituciones públicas, canalizado por una parte en actuaciones concretas que contribuyeron a mejorar el entorno necesario, tales como los trampolines tecnológicos lanzados en Cataluña y los programas Atlantis y Campus en Andalucía. Además, se promovió el desarrollo de las redes de business angels, sobresaliendo Cataluña en esta iniciativa. Desde el lado inversor, destaca la labor llevada a cabo por ENISA, entidad líder en la concesión de préstamos participativos en España que cuenta con varias líneas específicas de financiación del emprendimiento, como son la Línea de Jóvenes Emprendedores y la Línea de Emprendedores. También debe señalarse a CDTI como entidad financiadora de empresas con una larga trayectoria en España. Ambas han pasado de tener una actividad muy marginal a dinamizar intensamente el emprendimiento a través de la concesión de numerosos préstamos participativos y créditos blandos a la pyme en sus etapas iniciales, lo que sin duda ha contribuido a nutrir de financiación el ecosistema. En 2017, ambas entidades, junto con la actividad de otros organismos públicos regionales que también invierten en startups a través de programas específicos de préstamos participativos,

\footnotetext{
9 A efectos estadísticos, la actividad de los corporate ventures se incluye en las cifras de venture capital que publica ASCRI anualmente.
}

concedieron financiación por valor de 39.900.000 euros (-41 por 100 menos respecto a 2016) en un total de 422 inversiones (-34 por 100 frente a 2016).

- España cuenta con dos hubs de innovación (Barcelona y Madrid), lo que le ha llevado a posicionarse como uno de los países más atractivos y prometedores del panorama europeo. Entre las dos ciudades se concentra más del 80 por 100 de la inversión de venture capital que se realiza en España. Asimismo, la mayor parte de la actividad de los fondos internacionales de venture capital se produce exclusivamente en estas dos ciudades. Los factores que han impulsado al éxito de ambas ciudades radica en las excelentes universidades de ingeniería y de negocios, calidad de vida elevada que ayuda a atraer talento extranjero, profesionales altamente cualificados con un coste relativamente bajo si se compara con otras ciudades, o grandes eventos de carácter internacional en torno a la startup y la innovación como 4YFN (Barcelona) o South Summit (Madrid), que han alcanzado una dimensión internacional en tan solo seis años. Ambas ciudades cuentan ya con varios casos de éxito de startups, que es uno de los factores que desencadena el círculo virtuoso que permite la creación de un ecosistema tecnológico autosostenible (Privalia, Social Point, Ticketbis, AlienVault o Trovit son algunos ejemplos de startups ya desinvertidas en los últimos años, convirtiéndose en importantes empresas globales gracias a la financiación de venture capital). En el último estudio publicado por Atomico ${ }^{10}$, que analiza los ecosistemas europeos tecnológicos, destacan Madrid y Barcelona por ser de las principales ciudades para atraer talento, tal y como se puede ver en los Cuadros 2 y 3 .

\section{Impacto económico y social de las inversiones de venture capital}

El estudio recientemente publicado sobre el impacto económico y social de las inversiones de venture

\footnotetext{
10 https://2017.stateofeuropeantech.com/
} 


\begin{tabular}{|lll|}
\hline \multicolumn{3}{|c|}{ CUADRO 2 } \\
\multicolumn{3}{|c|}{ DESTINOS PREFERIDOS DE LOS } \\
\multicolumn{3}{|c}{ EMPRENDEDORES EUROPEOS PARA } \\
\hline EMPEZAR DE NUEVO \\
Categoría & Ciudad & Cambio categoría \\
& versus 2016 \\
1 & Londres & 1 \\
2 & Berlín & -1 \\
3 & Barcelona & 1 \\
4 & París & 9 \\
5 & Ámsterdam & -2 \\
6 & Dublín & 0 \\
7 & Estocolmo & 1 \\
8 & Lisboa & -3 \\
9 & Múnich & -1 \\
10 & Milán & 15 \\
& FUENTE: State of European Tech 2017. Atomico. \\
\hline
\end{tabular}

capital realizadas entre 2005 y $2013^{11}$ concluye en señalar el impacto positivo generado en las compañías respaldadas por la financiación de venture capital. En el Gráfico 6 se pueden ver los resultados de la evolución de los principales indicadores empresariales de las empresas participadas por el venture capital en España entre 2005 y 2015, en comparación con empresas similares que no dispusieron de este tipo de financiación (grupo de control). Este análisis muestra que en cada empresa participada por el venture capital el incremento medio del empleo, el resultado bruto, los activos tanto totales como inmateriales, así como las ventas fue más positivo que el que presentaron estas mismas variables en empresas comparables no financiadas por esta fuente de financiación. En concreto, el empleo medio creado por empresa en la muestra

\footnotetext{
11 Estudio elaborado por José Martí Pellón, Catedrático de Economía Financiera, Universidad Complutense de Madrid, y publicado por la Fundación ICO. El estudio completo, incluyendo su metodología, se puede consultar en http://www.fundacionico.es/
}

\begin{tabular}{|c|c|c|}
\hline \multicolumn{3}{|c|}{$\begin{array}{c}\text { PRINCIPALES HUBS PARA } \\
\text { DESARROLLADORES PROFESIONALES }\end{array}$} \\
\hline Categoría & Ciudad & \\
\hline 01 & Londres & 303.594 \\
\hline 02 & París & 181.659 \\
\hline 03 & Moscú & 144.488 \\
\hline 04 & Madrid & 104.102 \\
\hline 05 & Berlín & 93.517 \\
\hline 06 & Ámsterdam & 90.058 \\
\hline 07 & Múnich & 82.877 \\
\hline 08 & Varsovia & 77.318 \\
\hline 09 & Estocolmo & 62.594 \\
\hline 10 & Frankfurt & 62.004 \\
\hline 11 & Dublín & 61.260 \\
\hline 12 & Zúrich & 60.046 \\
\hline 13 & Budapest & 57.944 \\
\hline 14 & Barcelona & 57.607 \\
\hline 15 & Viena & 56.776 \\
\hline 16 & Bucarest & 53.758 \\
\hline 17 & Milán & 52.004 \\
\hline 18 & Copenhague & 51.420 \\
\hline 19 & Roma & 47.051 \\
\hline 20 & Hamburgo & 46.582 \\
\hline
\end{tabular}

FUENTE: State of European Tech 2017, Atomico.

apoyada por venture capital en el período analizado se incrementó en 11 trabajadores, frente a uno del grupo de control.

Los resultados positivos que se muestran en este estudio se explican por factores de creación de valor característicos de la inversión de capital privado que se añaden a la inyección de recursos: profesionalización de la gestión, estabilidad financiera, credibilidad frente a terceros, apertura a nuevos enfoques de negocio, mejor alineación de intereses entre propiedad y gestión, y experiencia en otros mercados y sectores, entre otros. 


\section{GRÁFICO 6}

\section{IMPACTO MEDIO POR EMPRESA HASTA EL AÑO 2015 EN COMPARACIÓN CON LAS NO FINANCIADAS POR VENTURE CAPITAL}

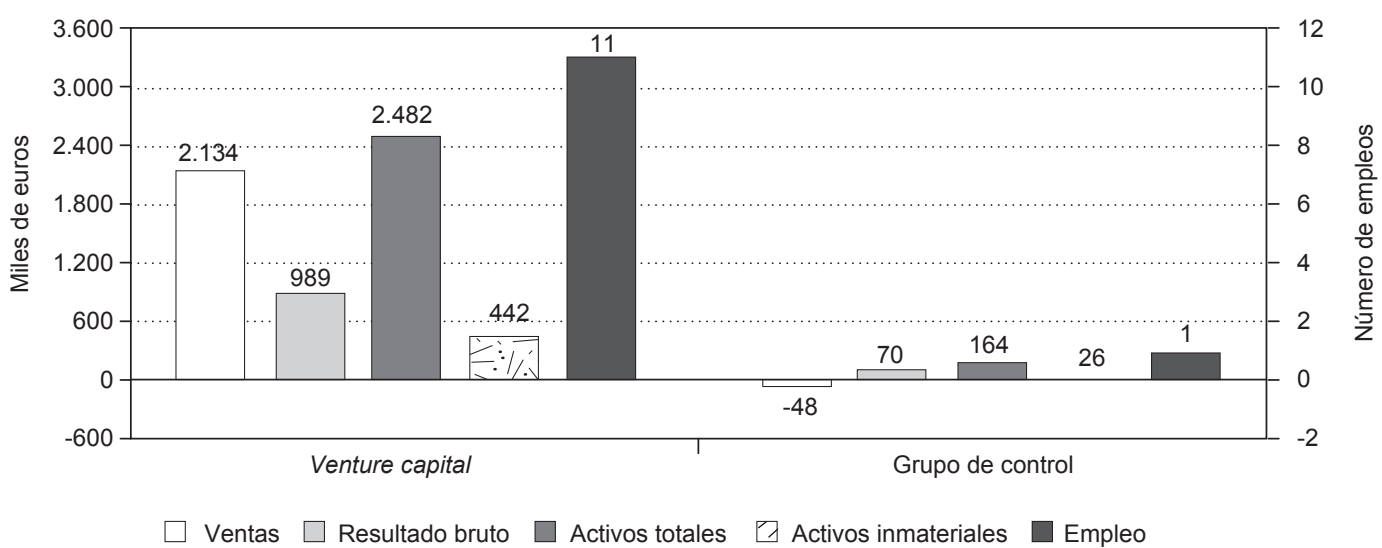

NOTA: Muestra formada por 1.035 empresas participadas por venture capital y sus respectivas comparables que no han contado con este tipo de financiación. Ambos conjuntos son similares en términos de distribución geográfica, sector económico y tamaño de las empresas. FUENTE: MARTÍ PELLÓN, J. (2018). Valoración del impacto económico y social de las inversiones de Venture Capital realizadas entre 2005 y 2013. Fundación ICO.

Respecto al impacto que implica el venture capital como asset class ${ }^{12}$ para los inversores en este tipo de fondos, los cuales incluyen fondos de pensiones, compañías de seguros, instituciones académicas, bancos, fundaciones privadas, fondos soberanos, fondos de fondos, family offices, grandes patrimonios, corporaciones e inversores particulares, su expectativa de retorno a largo plazo a la hora de invertir en este tipo de activos es superior a la esperada respecto a otras categorías de inversión más tradicionales como son la renta variable y renta fija, al estar asumiendo un mayor nivel de riesgo. Sin duda, la expectativa de retorno y el

\footnotetext{
12 Los fondos de venture capital, al igual que los de private equity, desde el punto de vista de activo de inversión (asset class), se estructuran a través de las aportaciones de inversores institucionales que conformarán el fondo de un importe determinado. La gestión del fondo la lleva a cabo un equipo de profesionales (general partners según terminología anglosajona), que asumen el compromiso de invertir estos recursos aportados por los inversores con mandatos temporales de inversión en empresas de un sector, fase y geografía determinado.
}

riesgo inherente a la inversión están ligados a la etapa en la que se encuentra la startup, siendo el riesgo menor cuando se trata de startups con ingresos, con modelos que ya tienen cierta tracción, y consecuentemente con una mayor visibilidad de salida de la inversión, frente a aquellas que se encuentran en fases muy iniciales de su actividad en las cuales el riesgo es considerablemente mayor y la ratio de proyectos fallidos elevada. En general, el retorno de la inversión de venture capital (al igual que con private equity) se compara normalmente con los índices del mercado de capitales como S\&P 500, Nasdaq, MSCI Europe etc., lo que permite poder tener una referencia de mercado. Según Prieto (2018), en un plazo de diez años de vida el retorno que tendría que tener el fondo de venture capital para compensar el excedente de riesgo en comparación a otros activos equivalentes debería estar en torno al 12 por 100 . Por el momento, no hay estadísticas relativas a los retornos de los fondos de 


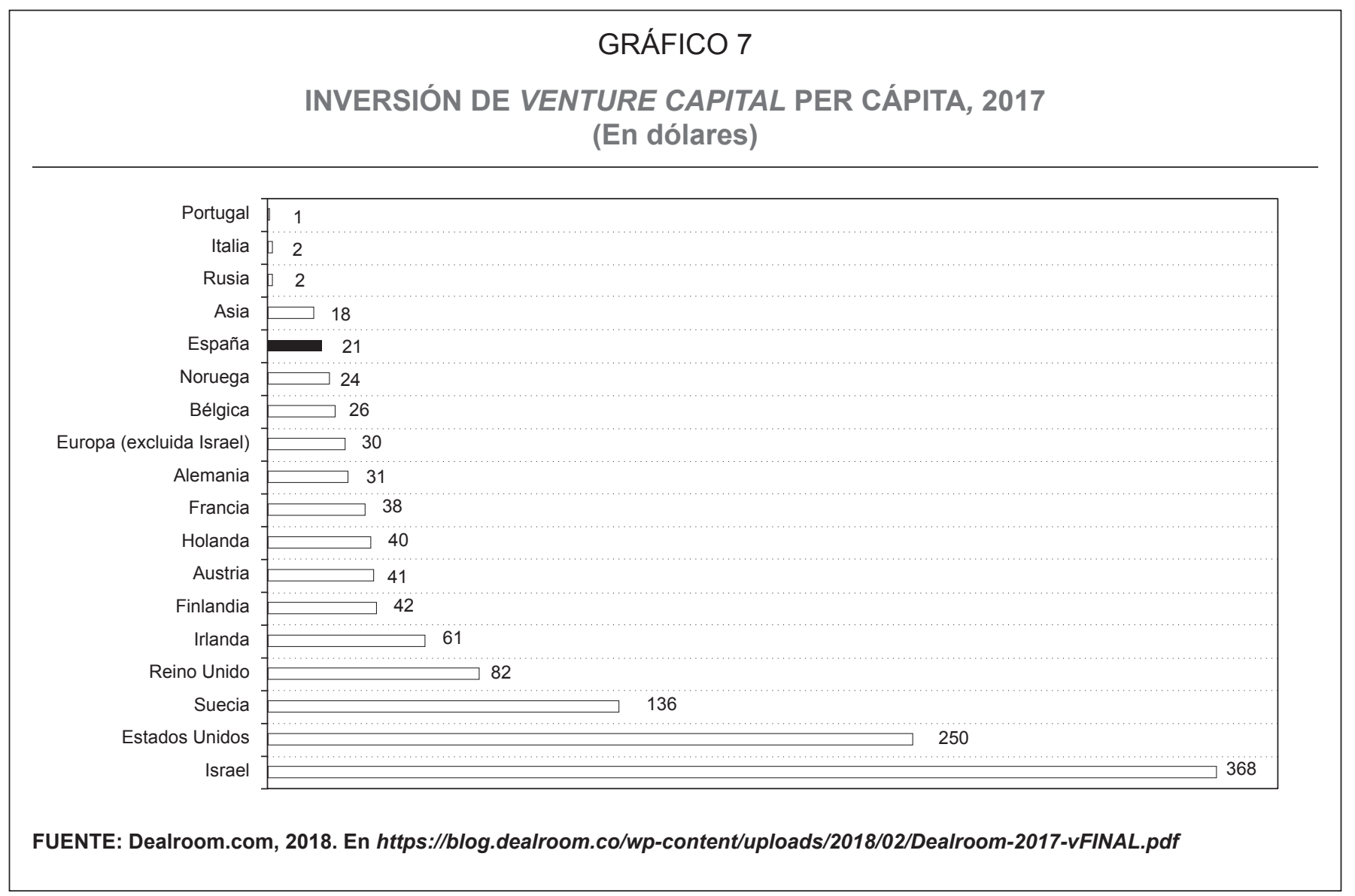

venture capital españoles, si bien la madurez que están alcanzando numerosos fondos del mercado español va a permitir iniciar esta línea de investigación a corto plazo.

Como aproximación para conocer cómo se suelen comportar los retornos de las empresas participadas por el venture capital, en un fondo se suelen distribuir en las siguientes proporciones: un tercio de la cartera de startups es pérdida total, otro tercio recupera el principal invertido en ellas, y es en el último tercio en el que se generan las plusvalías. Es decir, el éxito de un fondo depende de una o dos de sus participadas. Complementando esta estadística, Dave McClure, gestor del Fondo 500, apunta que entre el 50 por 100 y el 80 por 100 de las startups no generan ningún beneficio; entre el 15 por 100 y 25 por 100 alcanzan un múltiplo de $2 x$ a $5 x$ y tan solo entre el 5 por 100 y el 10 por 100 consiguen múltiplos de $10 x$ a $20 x^{13}$.

Volviendo a la positiva evolución del sector y su impacto en la pyme española, si comparamos el venture capital nacional con el resto de mercados, el mercado nacional está todavía muy lejos de los países líderes en esta actividad, como son Israel, Estados Unidos o Suecia, tal y como se aprecia en el Gráfico 7.

La diferente evolución de los países se explica, entre otros, por el importante papel que ha jugado la Administración en impulsar un régimen fiscal favorable para el desarrollo de esta actividad. En esta línea, son varios los retos que aún se tienen que afrontar y

13 TRENCHS, C. (2018). La madurez del mercado del venture capital. Una visión desde España. FUNCAS. 
medidas que se podrían implementar para ayudar a que la innovación tecnológica impulsada por el venture capital y su impacto en la creación de empleo y crecimiento económico aumenten en los próximos años:

- Lograr una mayor base de inversores institucionales españoles, tales como los fondos de pensiones, en los vehículos de venture capital y private equity. Es preciso estimular su participación con un mayor apoyo legislativo para que alcancen porcentajes de inversión comparables a los de sus competidores internacionales.

- Facilitar el acceso de «inversores sofisticados no profesionales» para que puedan participar en los fondos de venture capital. Actualmente, la rigidez de los criterios objetivos para calificar como «inversor profesional» (inversiones superiores a 100.000 euros) excluye de la posibilidad de invertir en fondos de venture capital a «inversores informados», cuyo capital es esencial para el desarrollo del tejido startup en España.

- Establecer incentivos fiscales a la inversión en vehículos de venture capital por parte de personas físicas. Para ello, se podría extender a las personas físicas la deducción del 20 por 100 en el IRPF sobre el capital invertido, tal y como ocurre con la deducción existente en el caso de inversiones particulares realizadas por business angels en empresas de nueva o reciente creación. Ayudar a canalizar el ahorro privado de forma ordenada y diversificada a este activo, en línea con lo que ya han puesto en marcha países como Francia o Reino Unido, sería un camino deseable con externalidades muy positivas para el conjunto de la economía.

- Reducir trabas a la atracción de talento extranjero, facilitando que posiciones que no son clave en términos de organigrama pero sí para el desarrollo de la startup (como son los de programador) puedan acceder al visado de emprendedor (fast track).

- Atraer a profesionales cualificados creando un marco fiscal favorable a través de la modificación de los límites y fiscalidad actuales en la entrega de acciones a los trabajadores.
- Reformar la figura del exit tax. Actualmente, un emprendedor que deba/quiera trasladar la residencia de su empresa a cualquier país no europeo sufrirá un gravamen sobre las plusvalías teóricas de sus participaciones, antes de que se produzca la posible plusvalía real por venta de las mismas. Más allá de que dicho gravamen desanima a emprendedores extranjeros a que desarrollen sus proyectos tecnológicos en nuestro país, el Tribunal de Justicia de la Unión Europea declaró un sistema tributario muy similar, como ilegal en Portugal.

\section{Conclusiones}

Durante los últimos años, el sector de venture capital ha experimentado un gran desarrollo en España hasta consolidarse como una de las propuestas más interesantes en el panorama europeo. Los factores que están detrás de este progreso, en el marco de un escenario de recuperación económica, se podrían resumir en los siguientes: aparición de nuevos actores en el ecosistema; mayores recursos para las gestoras nacionales; mayor experiencia y especialización en la gestión; creciente interés de los fondos internacionales en las startups españolas, debido a la madurez que están alcanzando muchas de ellas; mayores rondas de financiación, gracias en parte a los fondos internacionales; casos de éxito que empiezan a ser cada vez más numerosos; apoyo del sector público; y un cambio de mentalidad en la sociedad con mayor predisposición al emprendimiento.

A finales de 2017, había cerca de 3.000 millones de euros de inversión de capital riesgo repartidos en más de 1.700 pymes españolas. Tal y como vienen demostrando los estudios de impacto publicados, el capital privado, tanto en su papel de financiador de empresas maduras (private equity) como de empresas en primeras fases (venture capital), es una herramienta más para el desarrollo económico al ser capaz de crear valor en las empresas convirtiéndolas en compañías más grandes, productivas y eficaces. Sin embargo, y 
a pesar del camino recorrido y los importantes logros alcanzados, si se quiere que este sector de actividad siga contribuyendo a la generación de un ecosistema dinámico y generador de riqueza y empleo en nuestro país, es preciso que la Administración, tanto central como autonómica, encare con decisión los importantes retos que este sector afronta, para poder situarlo al mismo nivel que disfrutan los ecosistemas más modernos y permitir así a nuestras empresas competir en las mismas condiciones de los países más avanzados de nuestro entorno.

\section{Referencias bibliográficas}

[1] ALFÉREZ, A. (2013-2017). Informe de actividad venture capital \& private equity. ASCRI.
[2] MARTí PELLÓN, J.; ZUDAIRE J. y ALFEREZ, A. (2018). Evolución del venture capital y private equity en España y de su papel sobre la economía española. Funcas.

[3] MARTí PELLÓN, J. (2018). Valoración del impacto económico y social de las inversiones de venture capital realizadas entre 2013 y 2017. Fundación ICO.

[4] PRIETO, M. (2017). Los retornos del venture capital en Estados Unidos. Publicado en el blog TravelTechMedia: https://medium.com/traveltechmedia/los-retornos-del-venturecapital-en-estados-unidos-7497453e9536

[5] SALAS, M. (2018). El capital riesgo informal en España. 2018. Financiación de business angels, crowdfunding, aceleradoras, otros. Webcapitalriesgo.

[6] TRENCHS, C. (2018). La madurez del mercado del venture capital. Una visión desde España. Funcas.

[7] VARIOS AUTORES (2018). Annual European Venture Capital Report 2017. www.Dealroom.com

[8] WEHMEIER, T. (2017). State of European Tech 2017. Atomico. 